\title{
A Folate Receptor- $\alpha-$ Specific Ligand That Targets Cancer Tissue and Not Sites of Inflammation
}

\author{
Balasubramanian Vaitilingam, Venkatesh Chelvam, Sumith A. Kularatne, Scott Poh, Wilfredo Ayala-Lopez, \\ and Philip S. Low
}

Department of Chemistry, Purdue University, West Lafayette, Indiana

Folic acid has been frequently exploited to target attached drugs to cells that overexpress a folate receptor (FR). Unfortunately, folic acid and folate-linked drugs bind equally well to both major isoforms of the FR - that is, FR- $\alpha$, which is primarily expressed on malignant cells, and FR- $\beta$, which is upregulated on activated monocytes and macrophages. Because both major isoforms of FR can be expressed simultaneously in the same organism, folic acid cannot enable selective targeting of therapeutic and imaging agents to either tumor masses or sites of inflammation. In an effort to develop a targeting ligand that can selectively deliver attached imaging and therapeutic agents to tumor cells, we constructed a reduced and alkylated form of folic acid, $\mathrm{N}^{5}, \mathrm{~N}^{10}$-dimethyl tetrahydrofolate (DMTHF) that exhibits selectivity for FR- $\alpha$. Methods: DMTHF_99mTc was injected into mice bearing FR- $\alpha$-expressing tumor xenografts and imaged by $\gamma$-scintigraphy. The selectivity for FR- $\alpha$ over FR- $\beta$ in vivo was examined by $\gamma$-scintigraphic images of animal models of various inflammatory diseases such as apolipoprotein E-deficient mice with atherosclerosis, DBA/1 LacJ mice with induced arthritis, C57BL/6J mice with muscle injury, and BALB/C mice with both FR- $\alpha$ tumor and ulcerative colitis, by administration of equal doses of DMTHF_99mTc and EC20-99mTc. The uptake of radiochelates in various organs was quantified by biodistribution studies. DMTHF-near-infrared dye conjugate and DMTHF-Oregon green dye conjugates were synthesized and evaluated for FR$\alpha$ selectivity over FR- $\beta$ in rat peritoneal macrophages and human peripheral blood monocytes, respectively, by flow cytometry. Fluorescence-guided imaging was also performed using folate and DMTHF dye conjugates. Results: The new targeting ligand was found to bind malignant cells in mice with solid tumor xenografts but not peripheral blood monocytes or inflammatory macrophages in animal models of atherosclerosis, rheumatoid arthritis, muscle injury, or ulcerative colitis. Results from optical and radioimaging studies and biodistribution experiments confirm the differential specificity of this new ligand for malignant masses. Conclusion: The new targeting ligand DMTHF enables selective noninvasive imaging and therapy of tumor tissues in the presence of inflammation.

Key Words: folate receptor alpha specific ligand; optical and radioimaging of tumors; tumor-targeted therapy

J Nucl Med 2012; 53:1127-1134

DOI: 10.2967/jnumed.111.099390

\footnotetext{
Received Oct. 11, 2011; revision accepted Mar. 14, 2012.

For correspondence or reprints contact: Philip S. Low, Department of Chemistry,

Purdue University, 560 Oval Dr., West Lafayette, IN 47907.

E-mail: plow@purdue.edu

Published online Jun. 12, 2012.

COPYRIGHT $\odot 2012$ by the Society of Nuclear Medicine, Inc.
}

C ancer is a major cause of death in the United States, second only to heart disease (1), killing more than half a million Americans per year (2). Importantly, many cancerrelated deaths are thought to be preventable if better methods for early detection of the disease can be developed. Unfortunately, most methods for tumor imaging either lack the sensitivity to detect early malignant loci or do not distinguish between tumor masses and sites of inflammation (3). Thus, ${ }^{18} \mathrm{~F}-\mathrm{FDG}$ not only effectively images cancer but also accumulates at sites of infection and immune-mediated inflammation (4), creating confusion over whether an imaged mass derives from a tumor or inflamed tissue (e.g., rheumatoid arthritis, Crohn disease, ulcerative colitis, and asthma). Similarly, radiolabeled forms of choline, amino acids, vitamins, and nucleosides also accumulate in both malignant tissues and sites of inflammatory disease $(4,5)$.

Folate-linked radionuclides have been frequently exploited for the radioimaging of cancer tissues due in large part to the overexpression of folate receptor- $\alpha(F R-\alpha)$ on many malignant cell types (6-13). One such radioimaging agent is FolateScan (Endocyte, Inc.) (EC20), a ${ }^{99 \mathrm{~m} T c-}$ based radiopharmaceutical currently in phase III clinical trials for evaluation of folate receptor-positive tumors $(11,14)$. Importantly, EC20 has also proven to be effective for imaging inflammatory diseases, primarily because of the overexpression of FR- $\beta$ on activated macrophages (15-19). As with other PET and radioimaging agents, dual imaging of both cancer and inflammatory diseases can render differentiation of the 2 pathologies difficult. One obvious solution to this uncertainty would be to design a targeting ligand with selectivity for either FR- $\alpha$ or FR- $\beta$. Because literature references suggest that different forms of folic acid might exhibit different affinities for FR- $\alpha$ and FR- $\beta$ (20-22), we undertook a search for modified forms of folic acid that might associate preferentially with FR- $\alpha$ over FR- $\beta$. In this paper, we demonstrate that an $\mathrm{N}^{5}, \mathrm{~N}^{10}$-dimethylated derivative of tetrahydrofolic acid (DMTHF) displays the desired selectivity for FR- $\alpha$ over FR- $\beta$. We also show that a ${ }^{99 \mathrm{~m}} \mathrm{Tc}$-chelate conjugate of DMTHF allows for selective imaging of cancer masses in animals containing both solid tumors and multiple types of inflammatory disease. 


\section{MATERIALS AND METHODS}

\section{Cell Culture and Animal Husbandry}

KB and A549 cells were obtained from American Type Culture Collection. M109 cells were a kind gift from Alberto Gabizon. CHO- $\beta$ cells were a kind gift from Endocyte, Inc. The cell lines were grown continuously as a monolayer in folate-free RPMI medium containing $10 \%$ fetal calf serum and $1 \%$ penicillin and streptomycin antibiotic cocktail in a $5 \% \mathrm{CO}_{2}: 95 \%$ air-humidified atmosphere at $37^{\circ} \mathrm{C}$. M109 cells were regenerated as reported earlier (11).

All animal procedures were approved by the Purdue Animal Care and Use Committee in accordance with guidelines of the National Institutes of Health. Normal rodent diets were not used, because they contain excessive amounts of folic acid, which elevate serum folate levels significantly above normal physiologic concentrations. Rather, all animals were maintained on a folatedeficient diet (Teklad; Harlan Laboratories Inc.) for at least $3 \mathrm{wk}$ before each study to lower their serum folate levels into the physiologic range. Control animals were also maintained on a folate-deficient diet.

\section{Binding Affinity and Specificity of DMTHF-99mTc Conjugate}

$\mathrm{KB}$ or CHO- $\beta$ cells $(100,000$ cells per well in $500 \mu \mathrm{L})$ were plated into 24-well Falcon plates and allowed to form adherent monolayers $(\sim 70 \%$ confluent $)$ overnight. Spent medium in each well was replaced with fresh medium $(0.5 \mathrm{~mL})$ containing increasing concentrations of EC20-99m Tc or DMTHF-99m Tc in the presence or absence of a 100-fold molar excess of folic acid, and the cells were incubated for $1 \mathrm{~h}$ at $37^{\circ} \mathrm{C}$. Cells were washed with phosphate-buffered saline $(\mathrm{PBS})(3 \times 1.0 \mathrm{~mL})$, after which $0.25 \mathrm{M}$ aqueous $\mathrm{NaOH}(0.5 \mathrm{~mL})$ was added to dissolve the cells. After a 10-min incubation, $450 \mu \mathrm{L}$ from each well were transferred to polypropylene tubes and counted for radioactivity in a $\gamma$-counter (Packard Instrument Co.). The binding affinity, $K_{d}$, was calculated using GraphPad Prism 4 (GraphPad Software) by plotting cell-bound radioactivity versus the concentration of radiotracer added.

\section{Flow Cytometry Analysis of KB Cells and Rat Peritoneal Macrophages}

$\mathrm{KB}$ cells were allowed to form monolayers in a T75 Falcon flask for $48 \mathrm{~h}$ in folate-deficient RPMI 1640 culture medium. The confluent cells were trypsinized, and $1 \times 10^{6}$ cells were transferred into each Eppendorf tube and centrifuged. Spent medium was removed, and the pelleted cells were resuspended in fresh medium containing either DMTHF-DyLight680 (Pierce Thermo Scientfic) conjugate $(100 \mathrm{nM})$ or folate-DyLight680 conjugate $(100 \mathrm{nM})$, either in the presence or in the absence of a 100 -fold molar excess of free folic acid. After incubation for $1 \mathrm{~h}$ at $37^{\circ} \mathrm{C}$, the cells were washed 3 times with PBS $(0.5 \mathrm{~mL})$ and resuspended in fresh PBS $(0.4 \mathrm{~mL})$. Bound fluorescence was analyzed using a flow cytometer (Cytomics F500; Beckman Coulter).

For similar analysis of rat peritoneal macrophages, experimental adjuvant-induced arthritis was induced in Lewis rats according to a previously reported procedure (18). After $25 \mathrm{~d}$ of arthritis induction, the rats were sacrificed by $\mathrm{CO}_{2}$ asphyxiation. Peritoneal macrophages were harvested by injecting $60 \mathrm{~mL}$ of PBS into the peritoneal cavity and, after brief massage of the abdomen, removing the cells using the injection syringe (23). Peritoneal cells were transferred to Eppendorf tubes and washed and stained as described above, except $\mathrm{CD} 11 \mathrm{~b}^{+}$macrophages, which were identified by costaining with anti-CD11b-PE (phycoerythrin-labeled antibody to CD11b; Invitrogen).

\section{Flow Cytometry Analysis of Human Peripheral Blood Monocytes}

Human peripheral blood monocytes were isolated from whole human blood by a procedure described elsewhere (24). Briefly, whole human blood in anticoagulant was collected from healthy volunteers after obtaining informed consent, and the mononuclear cells were separated by Ficoll-Paque centrifugation (GE Healthcare). Cells were resuspended in Eppendorf tubes in folate-deficient RPMI 1640 culture medium containing either DMTHF-Oregon Green488 (Pierce Thermo Scientific) $(100 \mathrm{nM})$ or folate-Oregon Green488 (100 nM) plus CD11b Tri-Color (Life Technologies Corp.) to label human monocytes. For competition studies, a 100-fold molar excess of free folic acid was included in the suspension. After incubation for $1 \mathrm{~h}$ at $37^{\circ} \mathrm{C}$, the cells were washed 3 times with PBS $(0.5 \mathrm{~mL})$ and resuspended in fresh PBS $(0.4 \mathrm{~mL})$. Bound fluorescence was analyzed by flow cytometry as described above.

\section{Optical and Radioimaging of Animal Models of Cancer and Inflammatory Diseases}

Radioimages of murine models of human diseases were acquired with a Kodak Imaging Station coupled to a chargecoupled device camera operated with Kodak Molecular Imaging Software (version 4.0; Carestream Molecular Imaging). Abdomens were generally shielded with a lead plate to mask radioactivity emitted from the kidneys and bladder. The following parameters were used for radioimaging: acquisition time, $2 \mathrm{~min}$; f-stop, 4; focal plane, 7; field of view, 200; and binning, 4. For white light imaging, the parameters were the following: acquisition time, $0.05 \mathrm{~s} ; f$-stop, 11; focal plane, 7; field of view, 200; and no binning. For fluorescent imaging, abdomens were shielded with black construction paper to block fluorescence emitted from the kidneys and bladder. The parameters used were the following: acquisition time, $30 \mathrm{~s}$; excitation filter of $\lambda=625 \mathrm{~nm}$; emission filter of $\lambda=700 \mathrm{~nm}$; $f$-stop, 4; focal plane, 7; field of view, 200; and binning, 4 .

\section{Biodistribution of Radioimaging Agents}

After the radioimaging studies, animals were dissected and selected organs and tissues were collected into preweighed $\gamma$-counter tubes. Radioactivity of weighed tissues and test compounds were counted in a $\gamma$-counter. Counts per minute were decay-corrected, and results were calculated as percentage injected dose per gram of wet tissue (\% ID/g).

\section{Analysis of Tumor-Bearing Mice}

Six-week-old female $n u / n u$ mice (Harlan Laboratories Inc.) were inoculated subcutaneously on their shoulders with $\mathrm{KB}$ or A549 cells $\left(1.0 \times 10^{6}\right.$ cells/mouse in RPMI medium $)$ using a 25-gauge needle, as reported previously (25). Growth of the tumors was measured in 2 perpendicular directions every $2 \mathrm{~d}$ using a caliper, and the volumes of the tumors were calculated as $0.5 \times$ $\mathrm{L} \times \mathrm{W}^{2}$ ( $\mathrm{L}$ was the measurement of the longest axis, and $\mathrm{W}$ was the measurement of the axis perpendicular to $\mathrm{L}$ in millimeters). Radiotracer biodistribution studies were performed 12-15 d after tumor cell implantation, when the tumors reached approximately $50 \mathrm{~mm}^{3}$ in volume. The mice were randomly assigned to different 
treatment groups and injected intraperitoneally with DMTHF- ${ }^{99 m} \mathrm{Tc}$ (50 nmol, $5.55 \mathrm{MBq}[150 \mu \mathrm{Ci}]$ in $100 \mu \mathrm{L}$ of PBS). Four hours after injection, animals were sacrificed by $\mathrm{CO}_{2}$ asphyxiation, and imaging and biodistribution studies were performed as described above.

\section{Analysis of Atherosclerotic Mice}

B6.129-ApoE $E^{t m / U n c} / \mathrm{J}$ mice (apolipoprotein E-deficient $[a p o E-/-]$ ) breeding trios were purchased from the Jackson Laboratory and maintained in a temperature- and humidity-controlled environment with a 12-h dark-light cycle. Female mice were weaned at $3 \mathrm{wk}$ of age and maintained on either normal rodent chow or transferred at $5 \mathrm{wk}$ of age to a Western diet consisting of $2 \%$ cholesterol and $21.2 \%$ fat (TD.88137, Teklad; Harlan Laboratories Inc.) to accelerate the induction of atherosclerosis (26). After $16 \mathrm{wk}$ on a high-fat diet, the mice were randomly assigned to different treatment groups and treated (intraperitoneally) with EC20-99m Tc or DMTHF_99m Tc $(50 \mathrm{nmol}, 5.55 \mathrm{MBq}[150 \mu \mathrm{Ci}]$ in $100 \mu \mathrm{L}$ of PBS). Four hours after injection, animals were sacrificed by $\mathrm{CO}_{2}$ asphyxiation, and imaging and biodistribution studies were performed as described earlier.

\section{Analysis of Collagen-Induced Arthritic Mice}

Seven-week-old male DBA/1 LacJ mice were purchased from Harlan Laboratories Inc. and maintained on a folate-deficient diet (Teklad; Harlan Laboratories Inc.). Collagen-induced arthritis was initiated as described elsewhere (18). Arthritis scores were recorded by following the weighted criterion established by Chondrex, Inc. When the mice attained an arthritis score of 7 , they were randomly assigned to different treatment groups and injected with EC20-99m Tc or DMTHF-99m Tc $(50 \mathrm{nmol}$, $5.55 \mathrm{MBq}[150 \mu \mathrm{Ci}]$ in $100 \mu \mathrm{L}$ of PBS). Four hours after injection, animals were sacrificed by $\mathrm{CO}_{2}$ asphyxiation, and imaging and biodistribution studies were performed as described earlier.

\section{Analysis of Muscle Injury Model in Mice}

Six-week-old male C57BL6J mice were purchased from Harlan Laboratories Inc. and maintained on a folate-deficient diet (Teklad; Harlan Laboratories Inc.) for at least $3 \mathrm{wk}$ before analysis. Muscle injury was caused by injecting cardiotoxin from Naja atra, as reported elsewhere (27). Briefly, mice were anesthetized with $3 \%$ isoflurane, and the right tibialis anterior muscle of each mouse was injected with $100 \mu \mathrm{L}$ of cardiotoxin I (10 $\mu \mathrm{M}$; Sigma-Aldrich). Three days after injection, the mice were randomly assigned to different treatment groups and injected (intraperitoneally) with EC20-99m $\mathrm{Tc}$ or DMTHF- ${ }^{99 \mathrm{~m}} \mathrm{Tc}$ as described above.

\section{Analysis of Tumor-Bearing Mice That Were Simultaneously Induced to Develop Ulcerative Colitis}

Six-week-old female BALB/c mice were purchased from Harlan Laboratories and maintained on a folate-deficient diet (Teklad; Harlan Laboratories Inc.). After $1 \mathrm{wk}$ of acclimation, the mice were inoculated subcutaneously on the shoulder with $0.1 \mathrm{~mL}$ of M109 tumor cell suspension $\left(1 \times 10^{6}\right.$ cells) (11). Subcutaneous tumor growth was monitored daily, and at day 10 after tumor inoculation, $4 \%$ dextran sulfate sodium was added to their drinking water for $6 \mathrm{~d}$ to induce experimental ulcerative colitis (28). After colitis induction, the animals were randomly divided into different treatment groups and injected with EC20-99m Tc or DMTHF- ${ }^{99 \mathrm{~m}} \mathrm{Tc}$ as described above.

\section{Preparation of Animals for Optical Imaging}

Animals bearing tumors and inflammatory diseases were prepared as described above and injected intraperitoneally with DMTHF-DyLight680 or folate-DyLight680 conjugate (4 nmol in $100 \mu \mathrm{L}$ of PBS). Four hours after injection, animals were sacrificed by $\mathrm{CO}_{2}$ asphyxiation, and images were acquired with a Kodak Imaging Station using the same parameters described in the "Optical and Radioimaging of Animal Models of Cancer and Inflammatory Diseases" section. To evaluate accumulation of test compounds, animals were dissected, specific organs and tissues of interest were excised, selected regions of interest were analyzed in the Kodak Imaging Station.

\section{RESULTS}

\section{Analysis of Relative Affinities of Folate and DMTHF for FR- $\alpha$ and FR- $\beta$ In Vitro}

Unlike many other imaging modalities, for which selective visualization of malignant overinflamed tissues is difficult (e.g., ${ }^{18}$ F-FDG PET and ${ }^{11} \mathrm{C}$-choline), folate uptake by cancer cells and activated macrophages involves different isoforms of the same receptor (i.e., FR- $\alpha$ and FR- $\beta$, respectively). Because these 2 isoforms are only $76 \%$ homologous, slight structural differences between the 2 isoforms could allow for selective binding to one form over its homolog, enabling the development of a targeting agent that is specific for either malignant or inflammatory disease. Because different forms of reduced folates have been reported to exhibit different affinities for FR- $\alpha$ and FR- $\beta$ (20-22), we prepared several modified folates and examined their affinities for FR- $\alpha$ and FR- $\beta$. One compound, DMTHF, showed evidence of specificity for FR- $\alpha$ over FR- $\beta$ and was therefore examined further for its ability to selectively target cancer cells.

Initial analysis of the affinity of DMTHF for FR- $\alpha$ was conducted by incubating increasing concentrations of a radioactive conjugate of DMTHF, termed DMTHF- ${ }^{99 m} \mathrm{Tc}$ (Supplemental Scheme 1; supplemental materials are available online only at http://jnm.snmjournals.org), with KB cells, a human nasopharyngeal cancer cell line known to express FR- $\alpha$. The level of bound radioactivity was assessed by $\gamma$-counting. As shown in Figure $1 \mathrm{~A}, \mathrm{DMTHF}-{ }^{99 \mathrm{~m}} \mathrm{Tc}$ associated with $\mathrm{KB}$ cells with an apparent $\mathrm{K}_{\mathrm{d}}$ of $38 \mathrm{nM}$ in a manner that was quantitatively inhibited by a 100-fold molar excess of free folic acid. Because parallel binding studies with EC20 (an identical ${ }^{99 \mathrm{~m} T c-c h e l a t i n g ~ a g e n t ~ t a r-~}$ geted with folic acid) yielded a $\mathrm{K}_{\mathrm{d}}$ of $12 \mathrm{nM}$ (Fig. 1C), which was also competitive with excess free folic acid, we concluded that DMTHF-99m Tc and EC20 exhibit comparable affinities for human FR- $\alpha$.

Because FR- $\beta$-expressing monocytes and macrophages constitute only a small fraction of the total cell population in any tissue and FR- $\beta$-positive myeloid cells cannot be easily cultured in vitro (23), it was not possible to directly measure binding of DMTHF-99m Tc to a suspension of FR$\beta$-expressing monocytes or macrophages. Therefore, 2 distinct DMTHF dye conjugates were synthesized and used in conjunction with fluorescent antibodies specific for macro- 


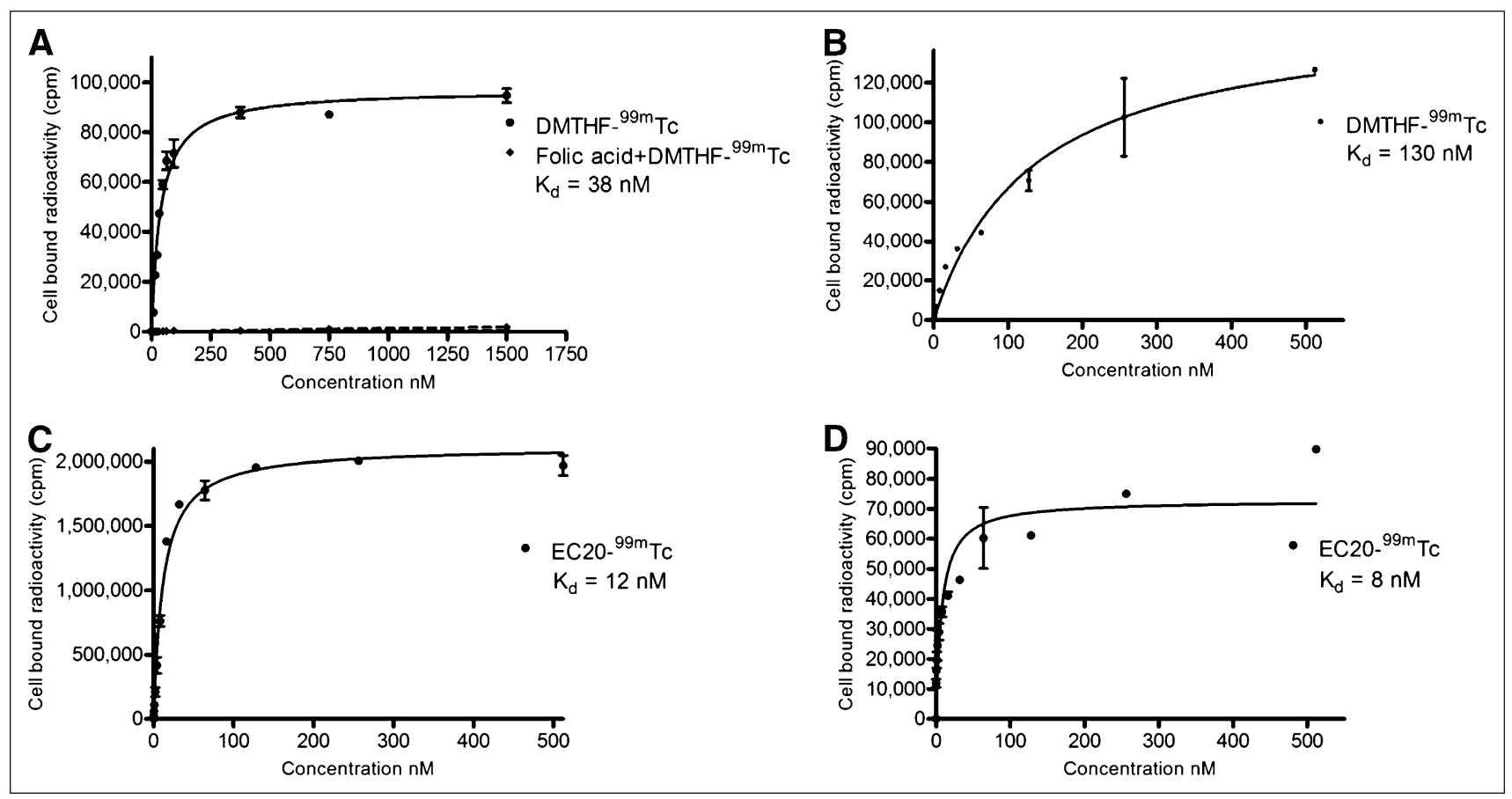

FIGURE 1. Binding affinity of DMTHF_99mTc for FR- $\alpha$-positive KB cells in culture in absence $(\bullet)$ and presence $(\bullet)$ of 100 -fold excess of free folic acid (A) ( $n=3$ ); DMTHF-99mTc for FR- $\beta$-positive CHO- $\beta$ cells $(\mathrm{B})(n=2)$; EC20-99mTc for FR- $\alpha-$ positive KB cells $(\mathrm{C})(n=3)$; and EC20-99mTc for FR- $\beta-$ positive CHO- $\beta$ cells (D) $(n=2)$. Error bars indicate SD.

phages to assess binding of DMTHF to FR-positive macrophages by flow cytometry. As seen in Supplemental Figure 1A, DMTHF-DyLight 680 conjugate binds avidly to FR$\alpha$-positive human KB cells, demonstrating that the conjugate can recognize the $\alpha$-isoform of FR. In contrast, DMTHF-DyLight 680 showed no affinity for CD11b ${ }^{+}$rat peritoneal macrophages (Supplemental Fig. 1B), even though the same cell population associated aggressively with folate-DyLight 680 (Supplemental Fig. 1C). These data demonstrate that DMTHF binds FR- $\alpha$ but displays little affinity for FR- $\beta$.
To confirm the absence of DMTHF affinity for human FR-positive, human peripheral blood was simultaneously labeled with the human monocyte marker, tricolor-labeled antihuman CD11b, and either DMTHF-Oregon Green488 or folate-Oregon Green488. As expected, folate-Oregon Green 488 conjugate caused a shift in the $\mathrm{CD}_{11} \mathrm{~b}^{+}$subpopulation of human peripheral blood leukocytes, whereas DMTHF-Oregon Green488 did not (Supplemental Figs. 1D and 1E), corroborating the aforementioned lack of affinity of DMTHF for FR- $\beta$-expressing myeloid cells.

FIGURE 2. Overlay of whole-body DMTHF-99mTc radioimages on white-light images of $n u / n u$ mice bearing FR- $\alpha$-positive KB tumor (A), FR- $\alpha$-positive KB tumor pretreated with excess folic acid to block all FR (B), and FR$\alpha$-negative A549 tumor (C). In images A-C, radioemissions from kidneys were blocked to permit easier visualization of conjugate distribution in other tissues. In SPECT/CT image of KB tumor-bearing nu/nu mouse (D), DMTHF-99mTc (11 MBq [300 $\mu \mathrm{Ci}])$ was injected $2 \mathrm{~h}$ before imaging on MiLabs $\mathrm{U}$ SPECT-II scanner.

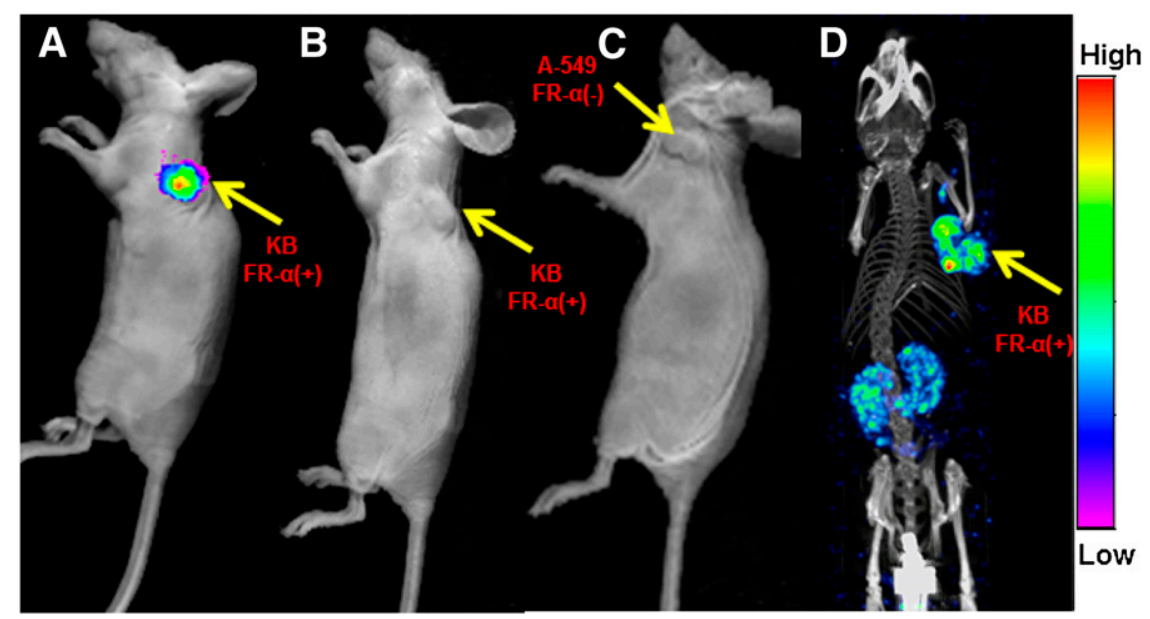




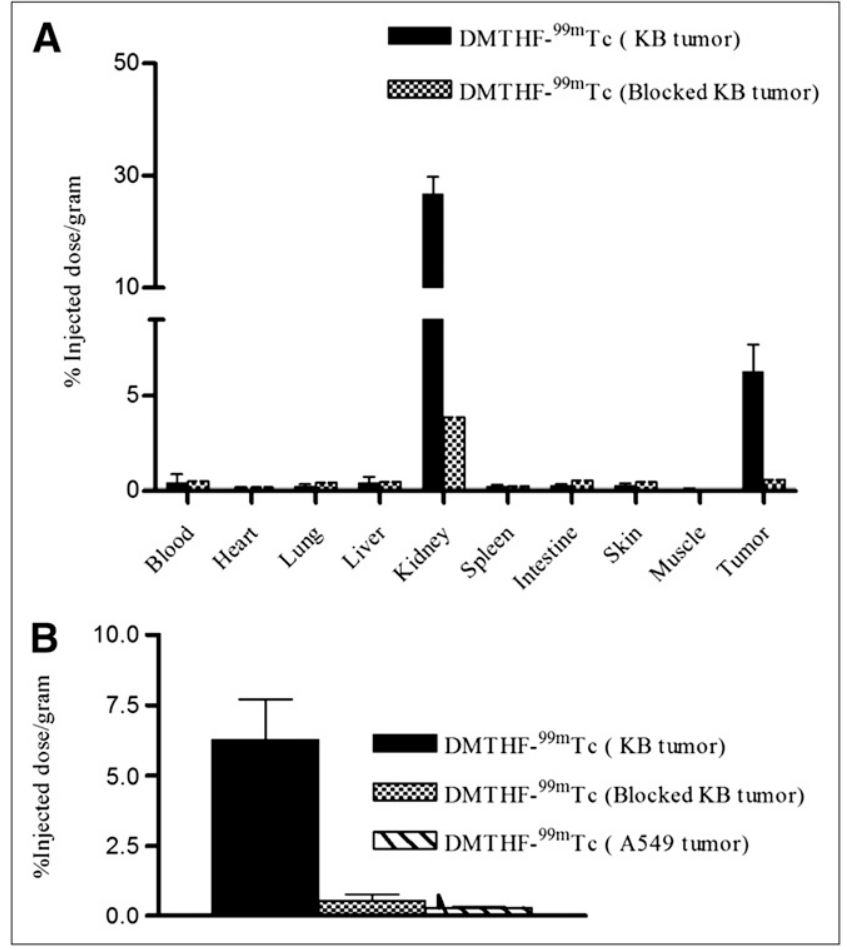

FIGURE 3. (A) Biodistribution of DMTHF- $99 \mathrm{mTC}$ in nu/nu mice bearing KB tumors in absence and presence of 100-fold excess of free folic acid. Error bars represent $S D$ ( $n=5$ mice per group). (B) Comparison of DMTHF_99mTc uptake by KB tumors, KB tumors with FR blocked by administration of 100 -fold excess of free folic acid, and A-549 tumors. Error bars represent SD ( $n=4$ mice/ group).

\section{Analysis of Relative Selectivities of Folate and DMTHF for FR- $\alpha$ and FR- $\beta$ In Vivo}

To evaluate the specificity of DMTHF for FR- $\alpha$-expressing tumors in vivo, we implanted both KB (an FR- $\alpha$-expressing tumor) and A549 (an FR- $\alpha$-negative tumor) xenografts on the shoulders of $n u / n u$ athymic nude mice and examined the uptake of DMTHF-99m Tc in both tumor models (Fig. 2). As seen in Figures $2 \mathrm{~A}$ and $2 \mathrm{C}$, the new targeting ligand displayed excellent specificity for the FR- $\alpha$-positive KB tumor but no affinity for the FR- $\alpha-$ negative A-549 tumor. The FR- $\alpha$ specificity of DMTHF-99mTc was further established in Figure 2B, where KB tumor uptake was blocked by preinjection of excess folic acid. A SPECT/CT image of another athymic $n u / n u$ mouse implanted with a $\mathrm{KB}$ tumor and injected intravenously with DMTHF- ${ }^{99 \mathrm{~m}} \mathrm{Tc}$ reveals the uptake in the tumor and kidneys against the background of the mouse's skeletal structure (Fig. 2D).

Quantitative confirmation of the above FR specificity was also provided by the biodistribution data in Figure 3A, where competitive binding was seen in the FR-positive tumor, but no significant binding was detected in the FRnegative tumor (Fig. 3B). Other than the tumor, only the kidneys, which also express FR- $\alpha$, showed significant ac-

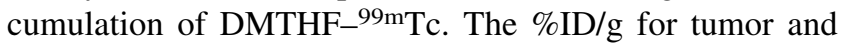
kidneys are 6 and 22, respectively. All other organs had significantly lower uptake, with less than $1 \% \mathrm{ID} / \mathrm{g}$, suggesting excellent tumor-to-nontumor tissue ratios for all organs except the kidneys: 72:1 (tumor to muscle), 50:1 (tumor to heart), 14:1 (tumor to liver), and 25:1 (tumor to spleen).

To further establish the selectivity of DMTHF- ${ }^{99 \mathrm{~m} T c}$ for FR- $\alpha$ over FR- $\beta$ in vivo, $\gamma$-scintigraphic images of animal models of various inflammatory diseases were compared after administration of equal doses of DMTHF-99mTc and EC20-99m Tc. ApoE-/- mice were selected for our first inflammation model, because these mice develop atherosclerotic plaques that are enriched in FR-positive macrophages (19). As seen in Figure 4A (right), mice injected with EC20-99m Tc displayed significant uptake in the aortacardiac region, where atherosclerotic plaque is most abun-
A

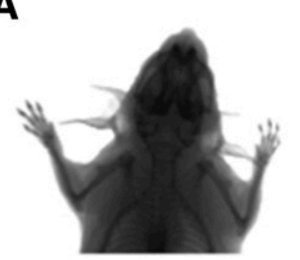

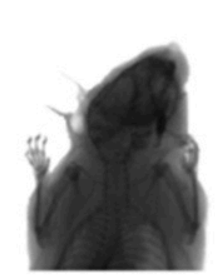

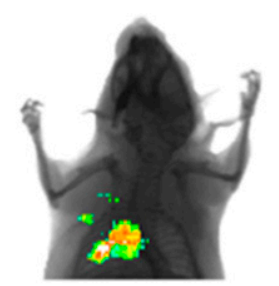

B

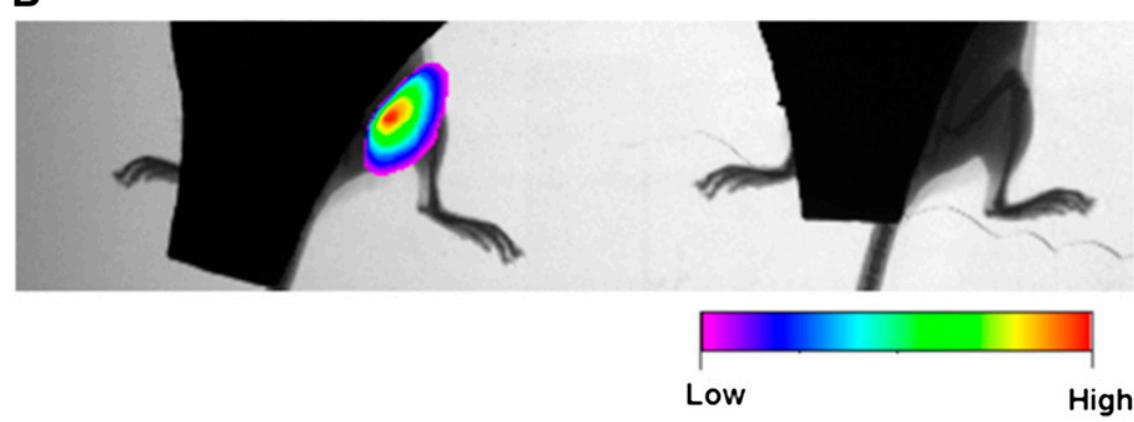

FIGURE 4. (A) Overlay of radioimages onto soft radiographic images of atherosclerotic apo $E^{-/-}$mice injected with $5.55 \mathrm{MBq}$ (150 $\mu \mathrm{Ci}$ ) of DMTHF_99mTc (left), 27.75 MBq (750 $\mu \mathrm{Ci})$ of DMTHF_99mTc (middle), or 5.55 MBq $(150 \mu \mathrm{Ci})$ of EC20-99mTc (right). (B) Overlay of radioimages onto soft radiographic images of C57BL/6J mice injected in hind leg with cardiotoxin to induce muscle trauma, and then imaged with $5.55 \mathrm{MBq}$ $(150 \mu \mathrm{Ci})$ of either EC20-99mTc (left) or DMTHF_99mTc (right). 
dant in this animal model. In contrast, mice injected with the same dose of DMTHF-99m Tc (Fig. 4A, left) displayed little or no uptake of radiotracer at the same site. In fact, even a 5-fold higher dose of DMTHF-99m Tc (Fig. 4A, middle) showed little evidence of accumulation in the atherosclerotic plaque. This absence of radioactive signal in mice treated with DMTHF- ${ }^{99 \mathrm{~m}} \mathrm{Tc}$ confirms the poor affinity of DMTHF- ${ }^{99 m}$ Tc for FR- $\beta$. This conclusion is also quantitatively established in the biodistribution analyses of Figure $5 \mathrm{~A}$, in which uptake of EC20- ${ }^{99 \mathrm{~m}} \mathrm{Tc}$ in the aortic arch is 10 -fold higher than uptake of DMTHF- ${ }^{99 \mathrm{~m} T c}$.

To further document the selectivity of DMTHF- ${ }^{99 \mathrm{~m}} \mathrm{Tc}$ for FR- $\alpha$, male DBA/1 LacJ mice were induced to develop experimental autoimmune arthritis using a commonly applied procedure for collagen-induced arthritis. Seven days after induction of inflammatory disease, animals were injected with either DMTHF- ${ }^{99 \mathrm{~m}} \mathrm{Tc}$ or $\mathrm{EC} 20-{ }^{99 \mathrm{~m}} \mathrm{Tc}$ and then imaged. As anticipated from previous studies (18), the collagen induction procedure was found to promote systemic inflammation throughout most tissues of the treated animals. More importantly, EC20-99m Tc but not DMTHF-99m Tc displayed elevated accumulation in both internal organs and inflamed joints of the arthritic animals (Fig. 5B). These data further support the poor affinity of DMTHF- ${ }^{99} \mathrm{~m}$ Tc for sites of inflammation.

Muscle injury as a consequence of physical trauma constitutes a common condition in which inflammation can interfere with normal tissue repair (29). To mimic this condition in a murine model, we injected the right tibialis anterior muscle of male C57BL/6J mice with cardiotoxin. The location and abundance of the resulting infiltrating activated macrophages were then examined $3 \mathrm{~d}$ after trauma by intravenous injection of either DMTHF- ${ }^{99 \mathrm{~m}} \mathrm{Tc}$ or EC20-99m Tc. As shown in Figure 4B (left), only mice treated with $\mathrm{EC} 20-99 \mathrm{~m} \mathrm{Tc}$ showed accumulation of radioactivity in the injured muscle, whereas DMTHF- ${ }^{99} \mathrm{~m}$ Tc-treated mice displayed little uptake of the radioactivity (Fig. 4B, right, and Fig. 5C).

To rigorously test the ability of DMTHF- ${ }^{99 \mathrm{~m}} \mathrm{Tc}$ to selectively image cancer tissue in the presence of inflamed tissues, an animal model was required that would present with

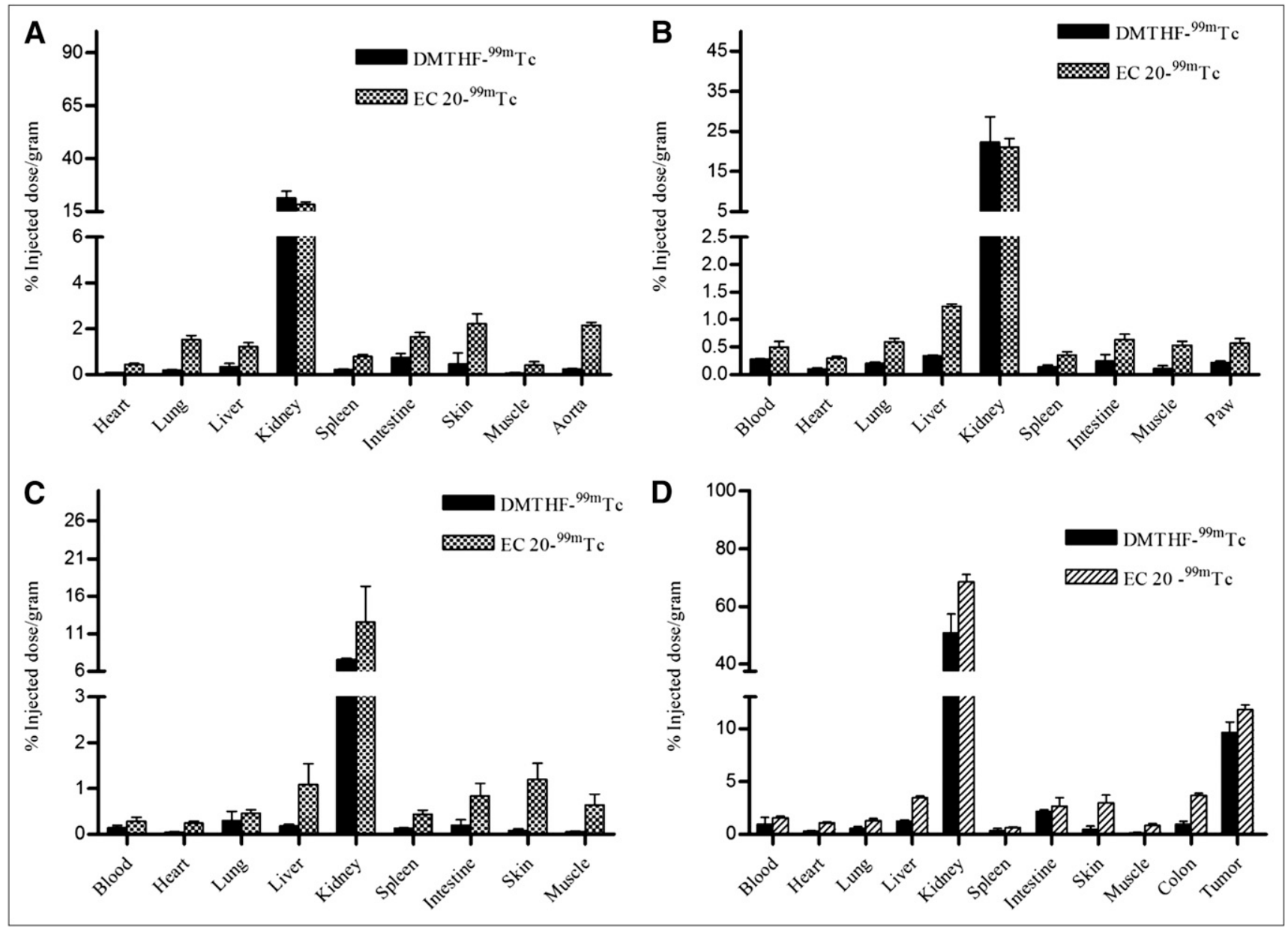

FIGURE 5. Biodistribution studies of EC20-99mTc and DMTHF_99mTc chelate in mice with various inflammatory or autoimmune diseases: apo $E^{-1-}$ atherosclerotic mice $(n=5)(\mathrm{A})$, DBA/1J mice treated to develop collagen-induced arthritis $(n=3)(\mathrm{B})$, C57BL/6J mice injected with cardiotoxin to develop muscle trauma $(n=5)(C)$, and M109 tumor-bearing BALB/C mice induced with DSS to develop ulcerative colitis $(n=5)(\mathrm{D})$. 
both malignant and inflamed tissues at the same time. For this purpose, male BALB/C mice were implanted with FR$\alpha$-expressing M109 tumor xenografts on their necks, and then treated with $4 \%$ dextran sodium sulfate (DSS) to induce ulcerative colitis 2 wk after tumor implantation. As seen in the Figure 6, tumor uptake of EC20-99mTc and DMTHF-99m Tc was similar, however, a significant difference in uptake of the 2 radioimaging agents was seen in the inflamed colons of the DSS-treated animals (Fig. 5D). The fact that DMTHF- ${ }^{99 \mathrm{~m}}$ Tc imaged primarily the tumor suggests that it can be used in vivo to distinguish sites of malignant disease from sites of inflammation.

Finally, to establish the generality of this selectivity of DMTHF for tumor over inflamed tissue, we elected to replace the radiolabeled payload with a fluorescent payload for use in fluorescence-guided imaging applications of both folate and DMTHF. Mice bearing M109 tumors were induced to develop ulcerative colitis as described above, and then injected with either DMTHF-DyLight680 or folate-DyLight680 before near infrared imaging. As seen in Supplemental Figure 2, dorsal images of both mice showed similar dye fluorescence in the dorsally implanted tumors. In contrast, ventral images of the same mice showed significant uptake only in the ulcerated gut of the folate-DyLight680treated mice. Indeed, image analysis of the relevant internal organs confirmed that although uptake of folate-Dylight680 (Supplemental Fig. 2D) remained visible in the tumor, kidneys, and ulcerated colon, accumulation of DMTHFDyLight680 was prominent only in the tumor and kidneys.

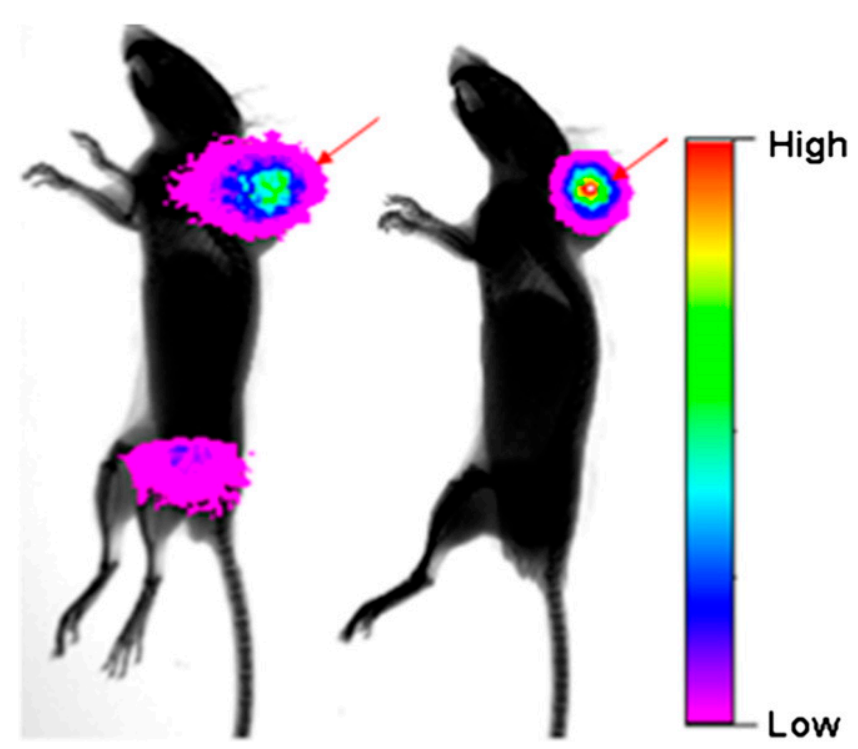

FIGURE 6. Overlay of radioimages onto soft radiographic images of M109 tumor-bearing BALB/c mice (arrows) induced with DSS to develop ulcerative colitis. Mice were injected intravenously with either EC20_99mTc (left) or DMTHF-99mTc (right) $4 \mathrm{~h}$ before radioimaging. Kidneys were shielded in both cases to allow better visualization of tumors and inflamed colon.

\section{DISCUSSION}

It is not uncommon for cancer patients to simultaneously be affected by one or more inflammatory or autoimmune diseases such as fibrosis, arthritis, hepatitis, ulcerative colitis, or pathogen infection. Although the inflammatory and malignant diseases may be located in distinct regions of the body, it is also possible that the 2 diseases could affect the same organ. Indeed, chronic inflammation is thought to be a common cause of cancers of the prostate, colon, liver, and esophagus (30). Though many tumor-targeted radioimaging agents have been developed to date, most simultaneously recognize inflamed tissues with similar affinity. This undesirable property is unfortunately also shared by all folate receptor-targeted imaging agents reported in the literature (31). In this paper, we have remedied this problem by identifying a modified FR ligand that displays significant specificity for FR- $\alpha$ over FR- $\beta$. This specificity should allow facile differentiation of proximal malignant from inflamed regions of the same organ or tissue. The improved specificity could prove useful when recurrence of cancer is suspected near the site of a prior surgery and standard imaging methodologies cannot distinguish between the inflammation caused by the surgery and any residual malignant disease (32). This specificity might also be beneficial to autoimmune disease patients who have undergone prolonged treatment with immunosuppressant. Because such patients may experience a higher incidence of cancer (33), an imaging agent that could reliably distinguish the 2 pathologies might be useful.

Finally, FR isoform selectivity not only might prove useful in disease diagnosis, but the ability to specifically target cancer cells with cytotoxic warheads might also reduce toxicity. Although many antimitotic drugs do not seem to harm FR- $\beta$-positive activated macrophages personal observations), other targeted therapies (e.g., radiotherapy, immunotherapy) will not likely discriminate between malignant cells and activated macrophages, killing both cell types with equal potency. In such cases, a tumor-selective targeting ligand might avoid aggravating an otherwise manageable inflammatory lesion. Thus, this new DMTH-targeting ligand with its preferential selectivity for FR- $\alpha$ may find multiple applications in the lab and in the clinic.

\section{CONCLUSION}

In this study, we have demonstrated the synthesis and application of a new targeting ligand that is selective for FR- $\alpha$. The radiopharmaceutical and fluorescent dye conjugates derived from this ligand can be used for selective noninvasive imaging of FR- $\alpha$-expressing tumors from the inflammatory microenvironment, distinguishing cancer from inflammation during diagnosis and evaluation of therapy. The ligand attached to cytotoxic drugs could open a new avenue to decrease offsite cytotoxicity that may arise during the treatment of cancer diseases by nontargeted drugs. 


\section{DISCLOSURE STATEMENT}

The costs of publication of this article were defrayed in part by the payment of page charges. Therefore, and solely to indicate this fact, this article is hereby marked "advertisement" in accordance with 18 USC section 1734.

\section{ACKNOWLEDGMENTS}

We thank Hari-Krishna R. Santhapuram and Le-Cun Xu, from Endocyte, Inc.; Xia Wei, Michael Hansen, Jiayin Shen, and Nancy L. Petretic for assisting with flow cytometry analysis; and the cell separation facility of the Bindley Bioscience Center, Purdue University. This work was supported by a grant from Endocyte, Inc. No other potential conflict of interest relevant to this article was reported.

\section{REFERENCES}

1. Centers for Disease Control and Prevention. Faststats. Available at: www.cdc. gov/nchs/fastats/lcod.htm. Accessed May 25, 2012.

2. Jemal A, Siegel R, Ward E. Cancer Statistics 2008. CA Cancer J Clin. 2008;58: 71-96.

3. Deane NG, Manning HC, Foutch AC, et al. Targeted imaging of colonic tumors in smad3-/- mice discriminates cancer and inflammation. Mol Cancer Res. 2007;5:341-349.

4. van Waarde A, Elsinga HP. Proliferation markers for the differential diagnosis of tumor and inflammation. Curr Pharm Des. 2008;14:3326-3339.

5. de Visser EK, Jonkers J. Towards understanding the role of cancer associated inflammation in chemoresistance. Curr Pharm Des. 2009;15:1844-1853.

6. Reddy JA, Low PS. Folate-mediated targeting of therapeutic and imaging agents to cancers. Crit Rev Ther Drug Carrier Syst. 1998;15:587-627.

7. Lu Y, Low PS. Folate mediated delivery of macromolecular anticancer therapeutic agents. Adv Drug Deliv Rev. 2002;54:675-693.

8. Lu Y, Sega E, Low PS. Folate receptor-targeted immunotherapy: induction of humoral and cellular immunity against hapten-decorated cancer cells. Int J Cancer. $2005 ; 116: 710-719$

9. Wang S, Low PS. Synthesis, purification, and tumor cell uptake of ${ }^{67} \mathrm{Ga}$-deferoxaminefolate, a potential radiopharmaceutical for tumor imaging. Bioconjug Chem. 1996; 7:56-62.

10. Mathias CJ, Low PS. Indium-111-DTPA-folate as a potential folate-receptortargeted radiopharmaceutical. J Nucl Med. 1998;39:1579-1585.

11. Leamon CP, Parker MA, Vlahov IR. Synthesis and biological evaluation of EC20: A new folate-derived, ${ }^{99 \mathrm{~m}} \mathrm{Tc}$-based radiopharmaceutical. Bioconjug Chem. 2002; 13:1200-1210.

12. Mathias CJ, Lewis MR, Low PS, Green MA. Preparation of ${ }^{66} \mathrm{Ga}$ and ${ }^{68} \mathrm{Ga}-$ labeled $\mathrm{Ga}(\mathrm{III})$-deferoxamine-folate as potential folate receptor targeted PET radiopharmaceuticals. Nucl Med Biol. 2003;30:725-731.

13. Siegel BA, Dehdashti F, Mathias CJ, Green MA. Evaluation of ${ }^{111}$ In-DTPAfolate as a receptor targeted diagnostic agent for ovarian cancer: initial clinical results. J Nucl Med. 2003;44:700-707.
14. Low PS, Kularatne SK. Folate targeted therapeutic and imaging agents for cancer. Curr Opin Chem Biol. 2009;13:256-262.

15. Low PS, Antony CA. Folate receptor-targeted drugs for cancer and inflammatory diseases. Adv Drug Deliv Rev. 2004;56:1055-1058.

16. Turk MJ, Breur GJ, Widmer WR. Folate-targeted imaging of activated macrophages in rats with adjuvant-induced arthritis. Arthritis Rheum. 2002;46:19471955.

17. Paulos CM, Turk MJ, Breur GJ, Low PS. Folate receptor-mediated targeting of therapeutic and imaging agents to activated macrophages in rheumatoid arthritis. Adv Drug Deliv Rev. 2004;56:1205-1217.

18. Paulos CM, Varghese B, Widmer WR, Breur GJ, Vlashi E, Low PS. Folatetargeted immunotherapy effectively treats established adjuvant and collagen-induced arthritis. Arthritis Res Ther. 2006;8:R77.

19. Ayala-López W, Xia W, Varghese B, Low PS. Imaging of atherosclerosis in apoliprotein E knockout mice: targeting of a folate-conjugated radiopharmaceutical to activated macrophages. J Nucl Med. 2010;51:768-774.

20. Wang X, Shen F, Freisheim JH, Gentry LE, Ratnam M. Differential stereo specificities and affinities of folate receptor isoforms for folate compounds and antifolates. Biochem Pharmacol. 1992;44:1898-1901.

21. Elnakat H, Ratnam M. Distribution, functionality and gene regulation of folate receptor isoforms: implications in targeted therapy. Adv Drug Deliv Rev. 2004; 56:1067-1084

22. Maziarz KM, Monaco HL, Shen F, Ratnam M. Complete mapping of divergent aminoacids responsible for differential ligand binding of folate receptors alpha and beta. J Biol Chem. 1999;274:11086-11091.

23. Xia W, Hilgenbrink AR, Matteson EL, Lockwood MB, Cheng JX, Low PS. A functional folate receptor is induced during macrophage activation and can be used to target drugs to activated macrophages. Blood. 2009;113:438-446.

24. He W, Wang H, Hartmann LC, Cheng JC, Low PS. In vivo quantitation of rare circulating tumor cells by multiphoton intravital flow cytometry. Proc Natl Acad Sci USA. 2007;104:11760-11765.

25. Mathias CJ, Wang S, Low PS, Waters DJ, Green MA. Receptor-mediated targeting of ${ }^{67} \mathrm{Ga}$-deferoxamine-folate to folate-receptor-positive human $\mathrm{KB}$ tumor xenografts. Nucl Med Biol. 1999;26:23-25.

26. Yamashita T, Kawashima S, Ozaki M, Yokoyama M. Propagermanium reduces atherosclerosis in apolipoprotein E knockout mice via inhibition of macrophage infiltration. Arterioscler Thromb Vasc Biol. 2002;22:969-974.

27. Yan Z, Choi S, Liu S, Zhang M, Williams RS. Highly coordinated gene regulation in mouse skeletal muscle regeneration. J Biol Chem. 2003;278:8826-8836.

28. Wirtz S, Neufert C, Weigmann B, Neurath MF. Chemically induced mouse models of intestinal inflammation. Nat Protoc. 2007;2:541-546.

29. Tidball JG. Inflammatory processes in muscle injury and repair. Am J Physiol Regul Integr Comp Physiol. 2005;288:R345-R353.

30. Ognjanovic S, Hainaut P. Inflammation in carcinogenesis. Compr Toxicol. 2010;401-415

31. Low PS, Henne WA, Doorneweerd DD. Discovery and development of folic-acid based receptor targeting for imaging and therapy of cancer and inflammatory disease. Acc Chem Res. 2008;41:120-129.

32. Dose-Schwarz J, Tiling R, Avril-Sassen S, et al. Assessment of residual tumor by FDG-PET: conventional imaging and clinical examination following primary chemotherapy of large and locally advanced breast cancer. Br J Cancer. 2010; 102:35-41.

33. Dantal J, Soulillou J-P. Immunosuppressive drugs and the risk of cancer after organ transplantation. N Engl J Med. 2005;352:1371-1373. 